Supporting Information

\title{
Polymerized Ionic Liquid-Containing Interpenetrating Network Solid Polymer Electrolytes for All-Solid-State Lithium Metal Batteries
}

Xiaowei Li, Yongwei Zheng, Qiwei Pan, Christopher Y. Li*

Department of Materials Science and Engineering, Drexel University, Philadelphia, PA 19104, United States

E-mail: chrisli@drexel.edu 

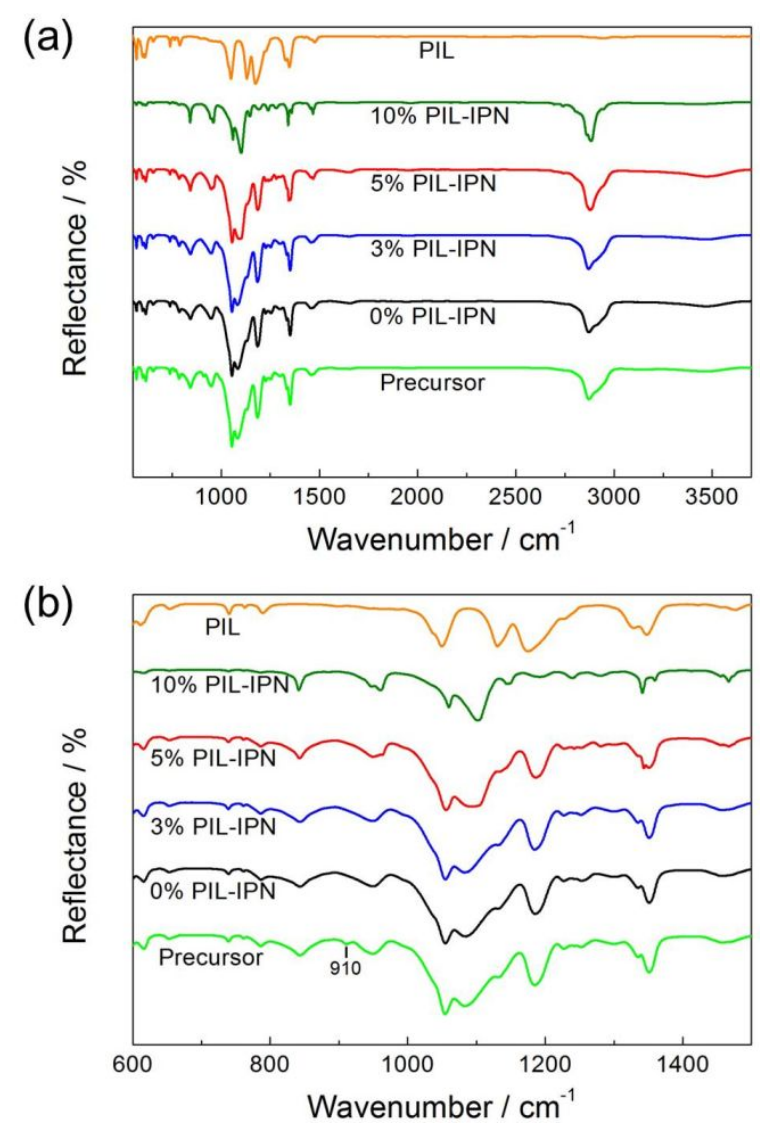

Figure S1. (a) FTIR spectra of the PIL, the precursor of POSS-4PEG2K and the PIL-IPN SPEs; (b) the enlarged spectra between 600 and $1500 \mathrm{~cm}^{-1}$.

As shown in the FTIR spectra, bands at around 2910, 2870, 1352 and $950 \mathrm{~cm}^{-1}$ are related to the $\mathrm{CH}_{2}$ on PEG chain. The band at $1476 \mathrm{~cm}^{-1}$ belongs to the P(DADMA) cation of PIL. The band at around $1100 \mathrm{~cm}^{-1}$ corresponds to the stretching of C-O-C of PEG chains and Si-O-Si of POSS. The bands of the TFSI anion are located at 1335, 1226, 1185, 1055, 785, 740 and $652 \mathrm{~cm}^{-1}$. The characteristic peak of the epoxy group at $910 \mathrm{~cm}^{-1}$ disappears in the four SPEs after cross-linking, indicating that most of the epoxy groups have reacted in the SPEs. 


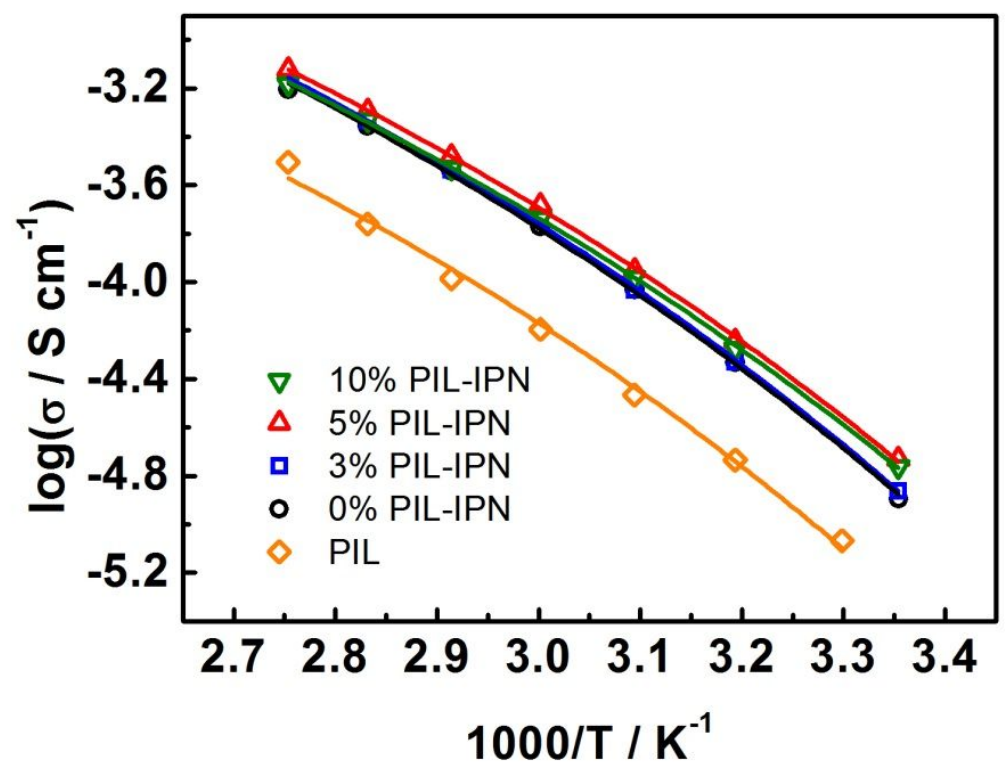

Figure S2. VTF fitting of the temperature dependent ionic conductivity.

Table S1. VTF fitting parameters of the SPE samples

\begin{tabular}{ccc}
\hline Sample & $\mathrm{A}\left(\mathrm{S} \mathrm{cm}^{-1} \mathrm{~K}^{1 / 2}\right)$ & $\mathrm{Ea}\left(\mathrm{kJ} \mathrm{mol}^{-1}\right)$ \\
\hline \% PIL-IPN & $21.3 \pm 2.2$ & $11.5 \pm 0.1$ \\
3\% PIL-IPN & $22.8 \pm 2.4$ & $11.5 \pm 0.1$ \\
5\% PIL-IPN & $16.4 \pm 1.1$ & $10.8 \pm 0.1$ \\
10\% PIL-IPN & $13.2 \pm 0.7$ & $10.6 \pm 0.1$ \\
PIL & $5.3 \pm 1.4$ & $10.1 \pm 0.3$ \\
\hline
\end{tabular}




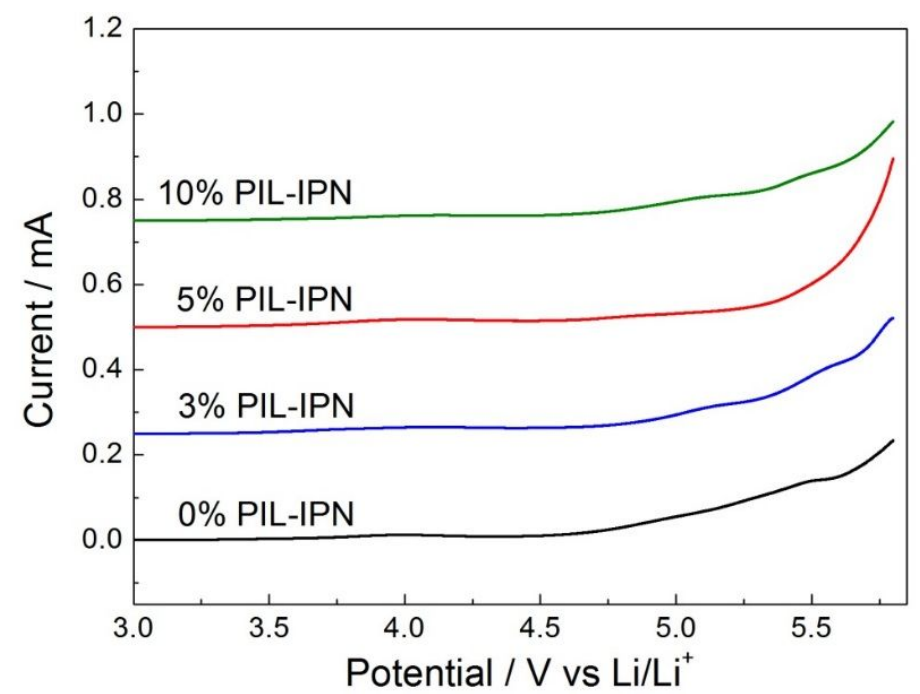

Figure S3. LSV curves of the PIL-IPN SPEs.
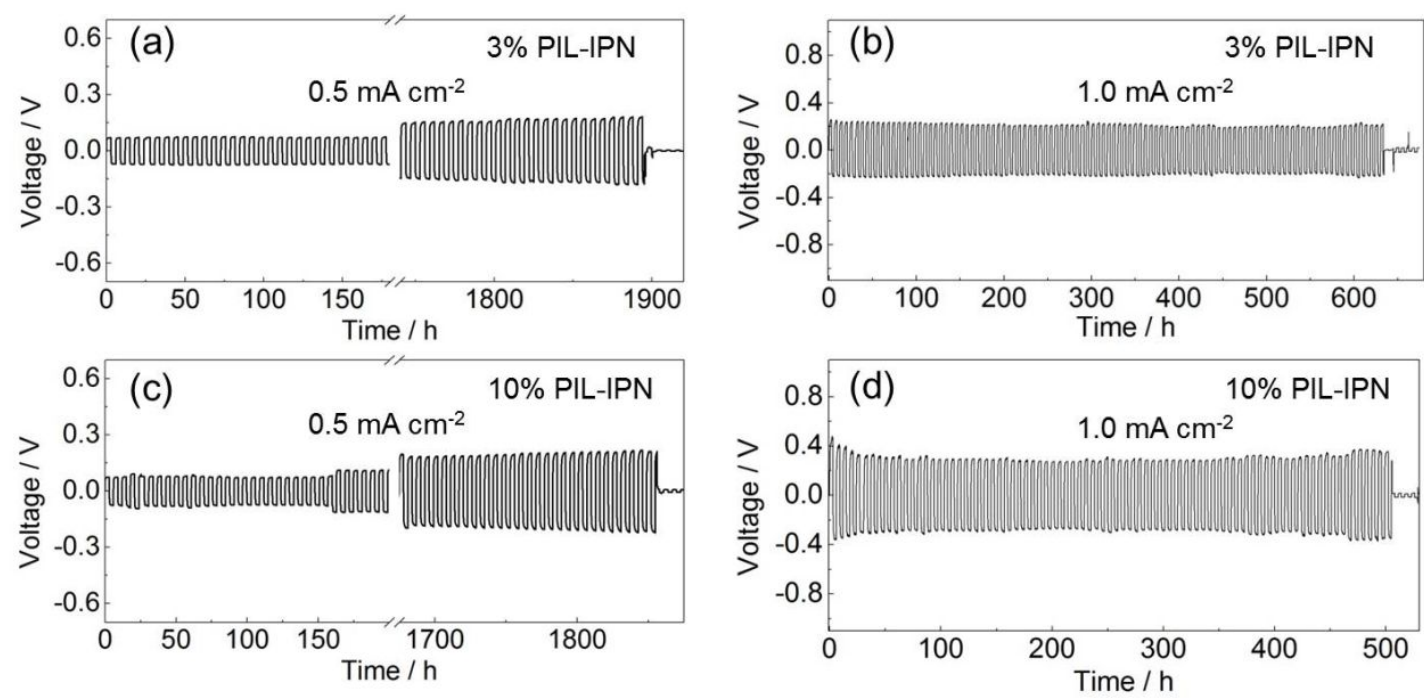

Figure S4. Time-dependent voltage profiles for symmetrical lithium cells containing the $3 \%$ and $10 \%$ PIL-IPN SPE samples at $90{ }^{\circ} \mathrm{C}$ with $3 \mathrm{~h}$ charge/discharge in each cycle under the current density of (a, c) $0.5 \mathrm{~mA} \mathrm{~cm}^{-2}$ and $(\mathrm{b}, \mathrm{d}) 1.0 \mathrm{~mA} \mathrm{~cm}^{-2}$. 

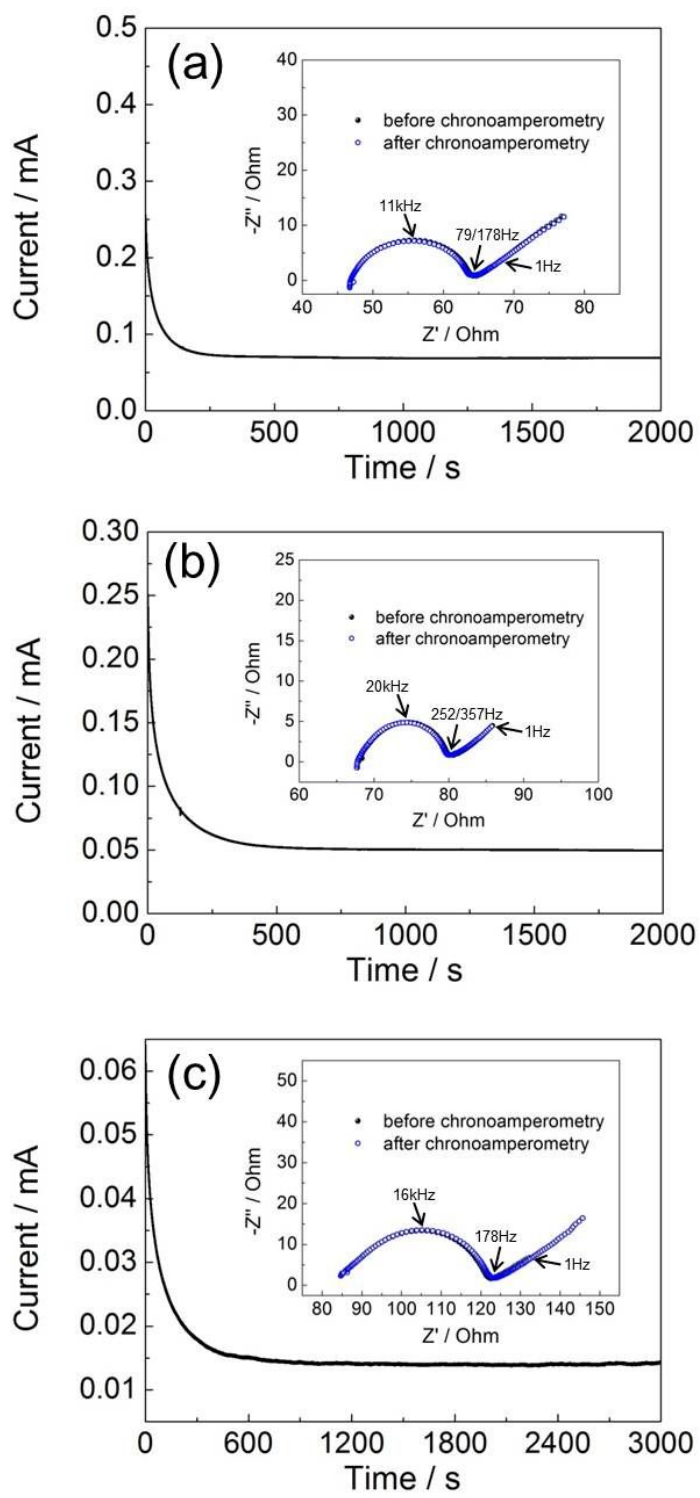

Figure S5. Chronoamperometry profiles and the impedance responses under initial and steady-state current conditions of the symmetrical lithium cells with (a) 3\% PIL-IPN, (b) 5\% PIL-IPN and (c) 10\% PIL-IPN sample at $90{ }^{\circ} \mathrm{C}$.

In the impedance spectra of symmetrical lithium cells shown in Figure S5, the real axis intercept at high frequency corresponds to the bulk of the electrolyte $R_{b}$. The depressed semicircle from high to middle frequency domain is attributed to the interphase resistence (SEI layer and charge transfer resistance) $R_{i}$. The spike at low frequency corresponds to Warburg resistence characteristic of the transport of charged particles. $^{[1]}$ 


\section{Short-circuit time prediction with the Chazalviel model}

According to the Chazalviel model, the dendrite growth velocity $v$ is equal to the drift velocity of anions in the applied electric field, so it is given by:

$$
v=\mu_{a} E_{0}
$$

where $\mu_{\mathrm{a}}$ is the anion mobility, the applied electric field $E_{0}=J / \sigma$, and $\sigma$ is the DC ionic conductivity. The time required for dendrites to grow, propagate and traverse the distance $L$ between electrodes is described by:

$$
t_{g}=\frac{\sigma L}{\mu_{a} J}
$$

Therefore, according to the model, the predicted short-circuit time $t_{\mathrm{sc}}$ will be:

$$
t_{s c}=\pi D\left(\frac{e C_{0}}{2 J t_{a}}\right)^{2}+\frac{\sigma L}{\mu_{a} J}
$$

The ambipolar diffusion coefficient $D$ could be calculated using the Nernst-Einstein equation

$$
D=\frac{\sigma k T}{C_{0} q^{2}}
$$

where $k$ is the Boltzmann constant, $T$ is the temperature, and $q$ is the diffusing species charge. To estimate the effective $\mathrm{C}_{0}$, we also assume LiTFSI is completely dissociated. The anion mobility $\mu_{\mathrm{a}}$ could be obtained from the Einstein relation as:

$$
\mu_{a}=\frac{q D t_{a}}{k T}
$$

References

[1] R. Bouchet, S. Lascaud, M. Rosso, J. Electrochem. Soc., 2003, 150, A1385. 